\title{
Análise de força e funcionalidade em paciente oncológico submetido ao criocongelamento: um relato de caso
}

\author{
Alessandra Cristina Amaral Rensi Silva 1, Francklin Trindade da Silva 1, Andreia Basta 1* \\ ${ }^{1}$ Serviço de Fisioterapia, Faculdade de Ciências Médicas da Santa Casa de São Paulo, São Paulo, SP, Brasil. \\ *Autor de correspondência: Andreia Basta. Rua Doutor Cesário Mota Junior, 112 - Vila Buarque. CEP: 01221-020 \\ - São Paulo, SP. Brasil. Telefone: (11) 97351-8997. Email: a.basta@globo.com.
}

Aprovação do Comitê de Ética em Pesquisa com Seres Humanos: Aprovado pelo comitê de ética em pesquisa com seres humanos da Faculdade de Ciências Médicas da Santa Casa de São Paulo (\#44455021.2.0000.5479).

Recebido em: Jan 9, 2022. Aceito em: Jan 26, 2022. Disponível online: Jan 27, 2022.

\section{Resumo}

Diversos tipos de abordagens cirúrgicas oncológicas são descritas como opções terapêuticas eficazes para o tratamento de diversos tipos de tumores primários, favorecendo a uma melhor chance de cura do paciente. A criocirurgia, compreendendo na utilização de nitrogênio líquido para para congelar e destruir o tecido tumoral, representa o tratamento padrão de tumores ósseos por obter uma menor taxa de recorrência local. Contudo, o impacto osteomuscular resulta em alterações funcionais pois a criocirurgia pode trazer comprometimentos vasculares e danos neurais à curto e longo prazo, podendo ocorrer uma grande incapacidade funcional, decorrente, por exemplo, de encurtamentos, rigidez articular, diminuição de força, fraturas, disfunções do sistema nervoso e possíveis quadros infecciosos. Nesta premissa, o objetivo deste estudo foi de avaliar a força muscular, a pisada, a funcionalidade e a qualidade de vida em um paciente submetido a cirurgia por criocongelamento para tumores ósseos malignos de fêmur distal e tíbia proximal.

Palavras-chaves: Criocirurgia; Funcionalidade; Incapacidade; Osteossarcoma.

\section{Introdução}

O câncer é um dos principais problemas de saúde no mundo com cerca de 18 milhões de novos casos apenas em 2018, estando entre as quatro principais causas de morte antes dos 70 anos de idade, com sua maior incidência no sexo masculino (cerca de 53\% de todos os novos casos) [1]. No Brasil, a estimativa para cada ano do triênio (2020-2022) aponta a ocorrência de 625 mil novos casos, com a maior distribuição na Região Sudeste, concentrando mais de $60 \%$ dos casos [2]. 
Análise da força e funcionalidade em paciente oncológico submetido ao criocongelamento

Em relação aos tumores ósseos, destaca-se o osteossarcoma, o tumor maligno primário mais comum, e o terceiro mais comum em crianças e adolescentes [3]. Geralmente seu surgimento está em áreas de crescimento ósseo principalmente localizados no fêmur distal (42\%), na tíbia proximal (19\%) e no úmero proximal $(10 \%)$.

Os principais sintomas incluem edema na região, diminuição da amplitude de movimento e sintomas respiratórios (uma vez que a metástase tumoral é a mais comumente encontrada nos pulmões). O prognóstico do paciente com osteossarcoma obteve melhoras nas últimas décadas, decorrente do avanço de técnicas cirúrgicas [4]. Uma opção terapêutica para o osteossarcoma consiste no tratamento cirúrgico que tem por objetivo a ressecção completa da doença por meio de uma ampla excisão do tumor [5].

A cirurgia contra o câncer é o tratamento primário para muitos tumores pois apresenta uma melhor chance de cura. O órgão ou o tecido deve ser removido por inteiro com uma margem de segurança (área livre da doença) por uma excisão de tecidos adjacentes para controle e cura local [6].

A aplicação bem-sucedida da técnica de criocirurgia depende da seleção apropriada do paciente, e da complexidade da técnica utilizada pelo cirurgião [7-9], sendo o tratamento padrão de tumores ósseos por obter uma menor taxa de recorrência local10.

O impacto osteomuscular resulta em alterações funcionais nos pacientes [11] pois a criocirurgia pode trazer comprometimentos vasculares e danos neurais à curto e longo prazo. Ao destruir as células tumorais, a criocirurgia também pode causas danos ao osso saudável circundante, aumentando o risco de fraturas no pósoperatório, e prejudicando a cicatrização óssea, podendo ser necessários procedimentos reconstrutivos para fornecer suporte mecânico adequado e evitar o acontecimento de fraturas [12]. O objetivo deste estudo foi avaliar a força muscular, a pisada, a funcionalidade e a qualidade de vida em um paciente submetido a cirurgia de criocongelamento para tumor ósseo maligno de tíbia proximal.

\section{Relato de Caso}

Paciente do sexo feminino, com 20 anos de idade, de etnia branca, solteira, com sobrepeso e sedentária, previamente hígida, apresentou-se ao serviço de emergência em um hospital público e terciário do estado de São Paulo, Brasil, com sintomas relacionados à quadro de joelho direito álgico persistente há 4 meses. Foi solicitado exames de imagem e anátomo patológico, sendo evidenciado o 
Análise da força e funcionalidade em paciente oncológico submetido ao criocongelamento

diagnóstico de Osteossarcoma pouco membro inferior direito (Figura 1).

diferenciado, proximal de tíbia no

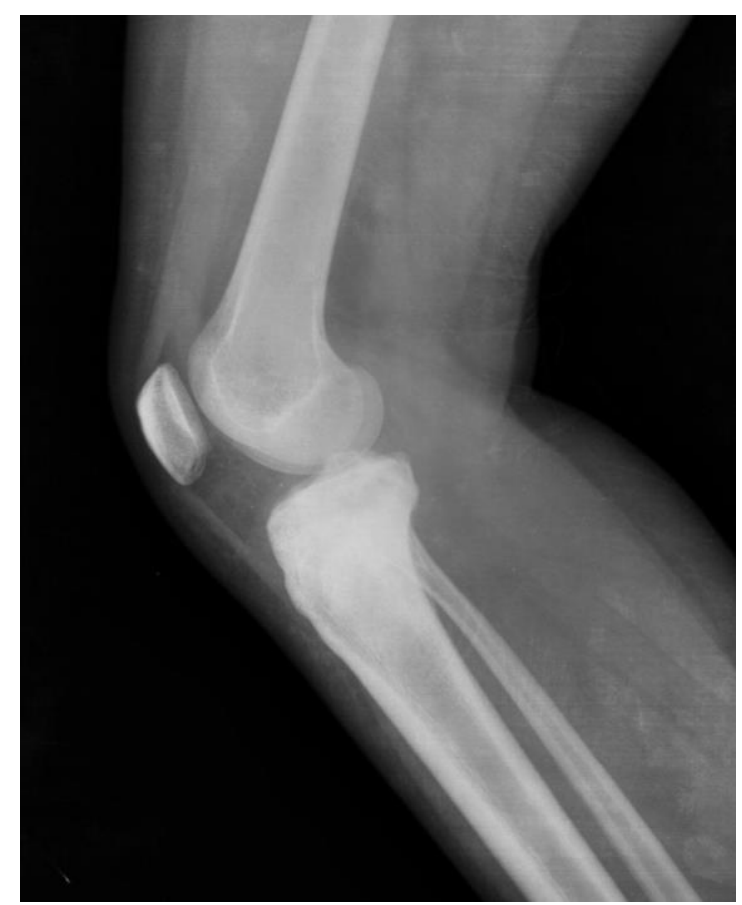

Figure 1. Raio -X de perna direita.

Como abordagens terapêuticas, o paciente foi submetido à 6 sessões de quimioterapia e a ressecção tumoral pela técnica de Criocongelamento com fixação por osteossíntese, sob acompanhamento da equipe de Fisioterapia Musculoesquelética por 2 vezes na semana, com duração média de 45 minutos cada sessão.

O tratamento com a paciente constituiu-se de liberação miofascial, mobilização articular, eletroestimulação neuromuscular, isometria, exercícios isotônicos concêntricos e excêntricos, exercícios de controle motor e treino de atividades funcionais com os objetivos de melhorar a amplitude de movimento articular, recrutar adequadamente fibras musculares diminuindo os efeitos da inibição artrogênica, fortalecer a musculatura, melhorar a função neuromuscular e promover atividade funcional adequada.

No contato com a equipe de fisioterapia musculoesquelética após 3 anos desde a realização da criocirurgia (Figura 2), foram realizadas uma série de avaliações funcionais a fim de verificar a eficiência do procedimento cirúrgico, tais como uma reavaliação de força e funcionalidade, através da dinamometria isométrica de flexão e extensão de joelho (Figuras 3) e extensão de quadril; uma análise da amplitude de 
movimento de extensão e flexão de joelho pela goniometria ativa; análise da baropodometria estática; análise da perimetria de coxa; avaliação funcional através do Escore Musculoskeletal
Tumor Society (MSTS) e por fim, uma avaliação da qualidade de vida através do Euro Quality of Life - 5 Dimensões (EQ5D).

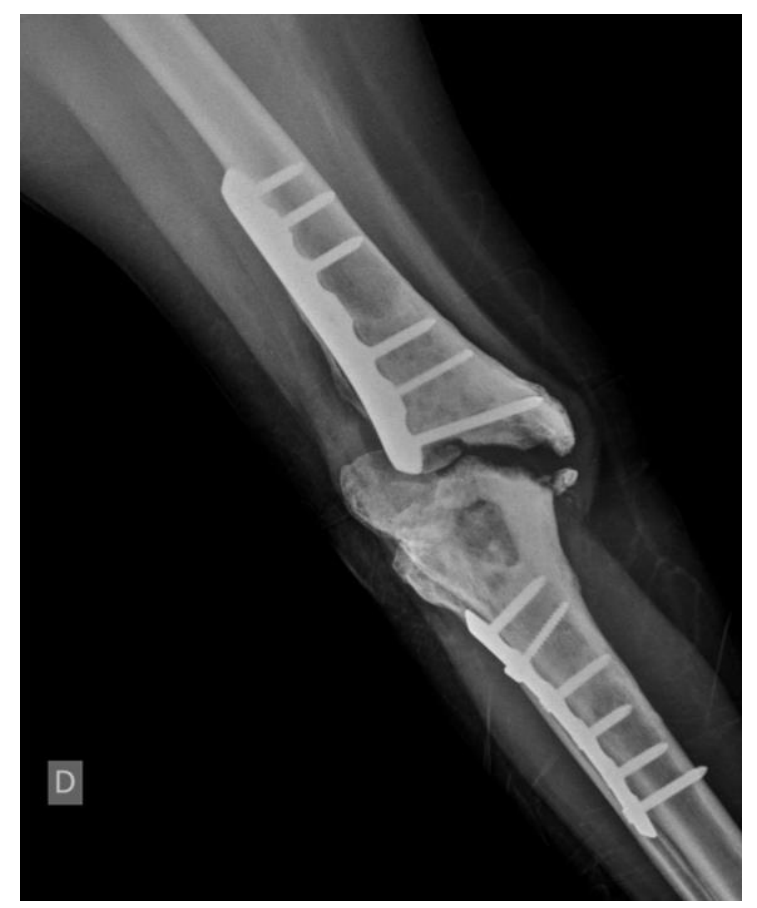

Figura 2. Raio-X de perna direita em dezembro de 2019.

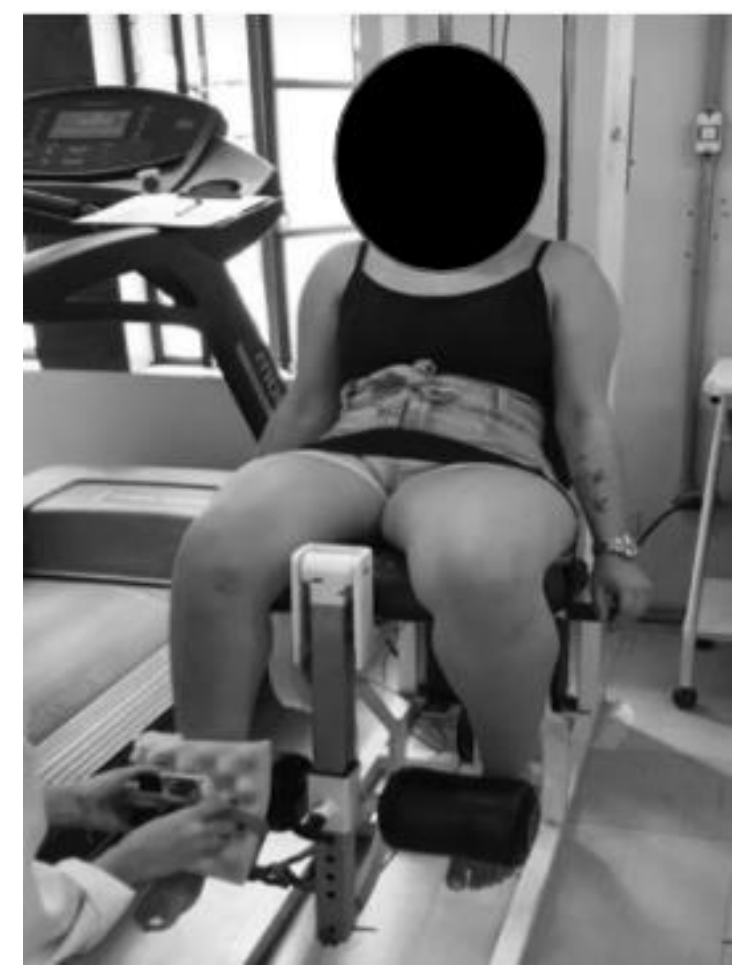

Figura 3. Dinamometria para extensão de joelho. 
Todos os aspectos clínicos da paciente foram demonstrados de forma descritiva nas tabelas 1 a 6 . Relacionado à análise de força, pôde-se observar, conforme mostrado na tabela 1 , que houve uma perda de força - comparado ao lado contralateral em que não houve acometimento tumoral - de aproximadamente $55 \%$ para flexão de joelho, 79\% para extensão de joelho, e em contrapartida um aumento de aproximadamente $35 \%$ de força para extensão de quadril.

Tabela 1. Dinamometria isométrica de joelho e quadril.

\begin{tabular}{c|c}
\hline Membro testado & Média Kg/f (+/-) \\
\hline Flexão de joelho direito & $4,67(0,76)$ \\
\hline Flexão de joelho esquerdo & $10,50(0.96)$ \\
\hline Extensão de joelho direito & $3,17(3,17)$ \\
\hline Extensão de joelho esquerdo & $15,40(1,65)$ \\
\hline Extensão de quadril direito & $12,80(1,61)$ \\
\hline Extensão de quadril esquerdo & $8,23(1,19)$ \\
\hline
\end{tabular}

Posteriormente, verificou-se que uma das principais limitações da paciente esteve relacionada com a rigidez articular, que pode ser observada na Tabela 2. Nota-se uma diminuição da amplitude de movimento, principalmente para a flexão de joelho, em comparação ao lado contralateral (Tabela 3).

Tabela 2. Amplitude de movimento ativo de joelho.

\begin{tabular}{c|c|c}
\hline Movimento & Direito & Esquerdo \\
\hline Extensão & $-8^{\circ}$ & $-2^{\circ}$ \\
\hline Flexão & $100^{\circ}$ & $135^{\circ}$ \\
\hline
\end{tabular}


Em relação ao controle postural através da análise pela baropodometria, evidenciado na tabela 3, obtivemos resultados importantes em relação à pressão plantar média e máxima exercida em diferentes situações, tais como base aberta ou fechada, mantendo os olhos abertos ou fechados durante 20 segundos. A pressão plantar média teve em seu resultado mais expressivo uma diferença de $21 \mathrm{Kpa}$ (Quilopascoal) durante o posicionamento em base fechada e olhos abertos, já a pressão plantar máxima teve seu resultado mais expressivo durante base fechada com olhos fechados, com uma diferença de $50 \mathrm{Kpa}$.

Tabela 3. Pico médio e máximo de pressão dos pés captado pela baropodometria.

\begin{tabular}{l|c|c|c|c}
\hline & $\begin{array}{c}\text { Pressão média } \\
\text { direito }\end{array}$ & $\begin{array}{c}\text { Pressão média } \\
\text { esquerdo }\end{array}$ & $\begin{array}{c}\text { Pressão } \\
\text { máxima direito }\end{array}$ & $\begin{array}{r}\text { Pressão máxima } \\
\text { esquerdo }\end{array}$ \\
\hline $\begin{array}{l}\text { Base aberta e olhos } \\
\text { abertos }\end{array}$ & $35.23 \mathrm{Kpa}$ & $43.40 \mathrm{Kpa}$ & $149.94 \mathrm{Kpa}$ & $156.63 \mathrm{Kpa}$ \\
\hline $\begin{array}{l}\text { Base fechada e } \\
\text { olhos abertos }\end{array}$ & $26.54 \mathrm{Kpa}$ & $47.54 \mathrm{Kpa}$ & $167.96 \mathrm{Kpa}$ & $182.74 \mathrm{Kpa}$ \\
\hline $\begin{array}{l}\text { Base aberta e olhos } \\
\text { fechados }\end{array}$ & $29.11 \mathrm{Kpa}$ & $39.13 \mathrm{Kpa}$ & $137.84 \mathrm{Kpa}$ & $179.57 \mathrm{Kpa}$ \\
\hline $\begin{array}{l}\text { Base fechada e } \\
\text { olhos fechados }\end{array}$ & $25.55 \mathrm{Kpa}$ & $42.58 \mathrm{Kpa}$ & $96.72 \mathrm{Kpa}$ & $146.75 \mathrm{Kpa}$ \\
\hline
\end{tabular}

De acordo com a perimetria realizada, podemos observar diminuição importante da massa muscular em 2,5 cm à $5 \mathrm{~cm}$ suprapatelar, $7 \mathrm{~cm}$ à $10 \mathrm{~cm}$ suprapatelar e $2,5 \mathrm{~cm}$ à $15 \mathrm{~cm}$ suprapatelar, conforme visto na tabela 4 .

A análise da qualidade de vida com base na aplicação do questionário EQ-5D (Tabela 5) nos mostrou que a paciente sofreu déficits de mobilidade, dificuldade moderada em atividades habituais, dor de moderada intensidade, além de moderada ansiedade e depressão. 
Tabela 4. Perimetria de coxas.

\begin{tabular}{|c|c|c|}
\hline Ponto de referência & Coxa direita & Coxa esquerda \\
\hline 5 cm suprapatelar & $40,0 \mathrm{~cm}$ & $42,5 \mathrm{~cm}$ \\
\hline $10 \mathrm{~cm}$ suprapatelar & $45,0 \mathrm{~cm}$ & $52,0 \mathrm{~cm}$ \\
\hline $15 \mathrm{~cm}$ suprapatelar & $54,0 \mathrm{~cm}$ & $56,5 \mathrm{~cm}$ \\
\hline
\end{tabular}

Tabela 5. Questionário EQ-5D.

\begin{tabular}{l|c}
\hline Mobilidade & 2 Pontos \\
\hline Cuidados Pessoais & 1 Ponto \\
\hline Atividades Habituais & 2 Pontos \\
\hline Dor & 2 Pontos \\
\hline Ansiedade / Depressão & 2 Pontos \\
\hline Mobilidade & 2 Pontos \\
\hline
\end{tabular}

Em relação à classificação funcional, segundo a Musculoskeletal Tumor Society (MSTS), verificou-se que a pontuação obtida foi de 11 pontos, equivalente à $36 \%$ da funcionalidade, conforme visto na Tabela 6.

Como visto em nosso estudo, a paciente apresenta diversas limitações que implicam diretamente em sua funcionalidade, a mesma encontra-se ainda em acompanhamento com a equipe médica mas houve uma descontinuidade do tratamento fisioterapêutico, pois há previsão de uma nova abordagem cirúrgica para colocação de uma endoprótese não convencional. 
Tabela 6. Classificação funcional segundo a MSTS.

\begin{tabular}{l|c}
\hline Dor & 2 Pontos \\
\hline Função & 5 Pontos \\
\hline Emocional & 1 Ponto \\
\hline Suporte & 0 Pontos \\
\hline Marcha & 3 Pontos \\
\hline Deformidade & 0 Pontos \\
\hline Pontuação Total & 11 Pontos \\
\hline
\end{tabular}

\section{Discussão e Conclusão}

Tendo em vista que o câncer é um dos principais problemas de saúde pública no mundo e que a estimativa de novos casos tenha uma maior incidência a cada dia, é de suma importância que os tratamentos sejam realizados de maneira adequada e efetiva para garantir uma boa qualidade de vida e funcionalidade do indivíduo.

Uma alta prevalência da perda de massa muscular está relacionada com a perda de força em pacientes oncológicos, conforme descrito por Aversa et al. [13], pacientes submetidos à quimioterapia apresentam uma maior perda de força muscular induzida por uma fadiga extrema, tendo então um agravante que contribui para que o paciente não realize exercícios físicos, o que acaba por exacerbar ainda mais a perda de massa muscular, tornando assim parte de um círculo vicioso.23 Dado esse que pode ser relacionado com a perda de massa muscular mostrada em nossa avaliação através da perimetria com uma diminuição significativamente importante encontrada na realização da dinamometria isométrica.

Em relação à amplitude de movimento, encontra-se na literatura um bom prognóstico após a ressecção tumoral, em estudo descrito por Higuchi et al. [14], após o tratamento com nitrogênio líquido para osteossarcoma de fêmur distal e tíbia proximal, realizado em 14 adolescentes com média de idade de 13 anos, a amplitude total de movimento para flexão de joelho pós operatória foi mínima de 90º sendo que 9 
Análise da força e funcionalidade em paciente oncológico submetido ao criocongelamento

participantes atingiram a amplitude de movimento total de flexão de joelho, estudo esse que entra em acordo com a apresentação clínica da nossa paciente, uma vez que a mesma apresenta flexão acima de $90^{\circ}$ porém com uma limitação acentuada quando comparado ao lado contralateral [14].

Segundo Rowe et al. [15], para uma funcionalidade satisfatória a amplitude mínima deve ser de 110º, uma vez que através de um estudo realizado com goniômetro eletrônico foi encontrado que a amplitude mínima de flexão de joelho para a marcha é de $90^{\circ}$, subir e descer escadas $90^{\circ}$ a $120^{\circ}$ e para atividades de vida diária como banho $135^{\circ}$, tendo como conclusão que $110^{\circ}$ corresponde a uma amplitude adequada para atividades de vida diárias [15].

Encontramos também na literatura uma associação do alto risco de queda em pacientes oncológicos decorrentes de uma alteração postural, assim como mostrado no estudo de Schmitt et al. [16] que através da estabilometria realizada em uma amostra de pacientes que sobreviveram ao câncer foi possível observar alterações posturais durante o ortostatismo, com estímulos proprioceptivos tais como manter os olhos abertos ou fechados16, assim como mostrado também no estudo de da Silva Alves et al. [17], realizado com mulheres mastectomizadas que existe uma diferença em relação a descarga de peso durante o apoio bipodal devido à uma mudança no centro de massa em relação ao lado acometido com o lado não acometido, ambos estudos corroboram com os achados da baropodometria realizada em nossa avaliação.

Em decorrência de múltiplas alterações, tais como diminuição da amplitude de movimento, diminuição da força e alterações posturais, a marcha apresenta-se alterada, assim como mostrado no estudo de Matsusaka [18] feito em 17 pacientes com histórico de osteossarcoma primário, através da análise de marcha feita por uma plataforma de força foi levado em consideração a força de reação ao solo, cadência, velocidade da marcha e variações do centro de massa, sendo encontrada alterações significativas principalmente uma diminuição da força de reação ao solo.

Em relação à qualidade de vida, deve-se primeiramente entender que os fatores descritos e mensurados estão em acordo com a individualidade biológica do paciente em todas as áreas da vida levando em consideração o impacto da doença [19]. Para pacientes oncológicos o desfecho em relação à funcionalidade encontra-se muitas vezes contraditório, uma vez que é possível encontrar resultados satisfatórios tais como no estudo supracitado de Higuchi et al. [14] em que os pacientes apresentaram uma pontuação média de $90 \%$ de 
Análise da força e funcionalidade em paciente oncológico submetido ao criocongelamento

funcionalidade com base no podem ser encontrados resultados

questionário do MSTS, assim como é possível encontrar resultados menos satisfatórios, tal como demonstrado por Huang et al. [20], que em estudo feito com 69 pacientes avaliados pelo MSTS 6 meses após a cirurgia, demonstraram uma pontuação média de 21 pontos, valor este mais próximo do que foi encontrado em nossa paciente, que apresentou 11 pontos, sendo correspondente à $36 \%$ de funcionalidade.

Uma vez que assim como descrito por Nayak et al. [21] a sintomatologia da doença tem uma grande influência na qualidade de vida entre os pacientes oncológicos, não havendo necessariamente algo que possa ser feito para o controle do impacto dos sintomas na qualidade de vida, mas sim um manejo adequado dos mesmos e na compreensão acerca da condição de vida apresentada pelo paciente visando a busca pela melhora funcional e dos objetivos acerca do tratamento.

Estudos encontrados na literatura demonstram um prognóstico com satisfatório grau de mobilidade articular, e uma esperada perda de massa muscular, o que diretamente acarreta perda de força muscular, alterações de estabilidade estática como alterações de descarga de peso e alterações no centro de massa $[13,16$, 20], e mesmo que ainda existam fatores conflitantes em relação à funcionalidade satisfatórios ou deficitários que irão afetar diretamente a qualidade de vida apresentada pela paciente, como pode ser visto no nosso estudo.

O acompanhamento multidisciplinar do serviço de Oncologia Ortopédica junto à equipe de fisioterapia citado no presente estudo contribuiu para um resultado satisfatório na retirada do tumor da paciente, apesar de ainda ser encontrado déficits na funcionalidade da paciente que devem ser trabalhadas junto à equipe de fisioterapia musculoesquelética.

Dentro das limitações do estudo encontra-se a descontinuidade do tratamento fisioterapêutico devido a questões médicas, não sendo possível acompanhar a longo prazo o estado de funcionalidade da paciente, além de ter sido realizado o estudo de um único caso para inferir implicações funcionais de uma técnica cirúrgica amplamente utilizada na oncologia ortopédica.

\section{Referências}

[1] Bray F, Ferlay J, Soerjomataram I, Siegel RL, Torre LA, Jemal A. Global cancer statistics 2018: GLOBOCAN estimates of incidence and mortality worldwide for 36 cancers in 185 countries. CA Cancer J Clin. 2018 Nov;68(6):394-424. 
[2] Ministério da Saúde. Instituto

Nacional do Câncer José Alencar Gomes da Silva. Estimativa 2020 - Incidência de Câncer no Brasil. Acesso em: https://www.inca.gov.br/sites/ufu.sti.inc a.local/files/media/document/estimativa -2020-incidencia-de-cancer-nobrasil.pdf).

[3] Simpson E, Brown HL. Understanding osteosarcomas. JAAPA. 2018 Aug;31(8):15-19. doi: 10.1097/01.JAA.0000541477.24116.8d.

[4] Haddox CL, Han G, Anijar L, Binitie O, Letson GD, Bui MM, Reed DR. Osteosarcoma in pediatric patients and young adults: a single institution retrospective review of presentation, therapy, and outcome. Sarcoma. 2014;2014:402509.

[5] Misaghi A, Goldin A, Awad M, Kulidjian AA. Osteosarcoma: a comprehensive review. SICOT J. 2018;4:12. doi: 10.1051/sicotj/2017028.

[6] Meller I, Weinbroum A, Bickels J, Dadia S, Nirkin A, Merimsky O, Issakov J, Flusser G, Marouani N, Cohen N, Kollender Y. Fifteen years of bone tumor cryosurgery: a single-center experience of 440 procedures and long-term followup. Eur J Surg Oncol. 2008 Aug;34(8):921-927.

[7] Ramming KP. Cryosurgery: the coming of the surgical ice age? J Surg
Oncol 1995;58(3):147-8.

doi: 10.1002/jso.2930580302.

[8] Erinjeri JP, Clark TW. Cryoablation: mechanism of action and devices. J Vasc Interv Radiol 2010;21(8 Suppl):S187-91. doi: 10.1016/j.jvir.2009.12.403.

[9] Usatoff V, Habib NA. Update of laser-induced thermotherapy for liver tumors. Hepatogastroenterology 2001;48(38):330-2.

[10] van der Geest IC, van Noort MP, Schreuder HW, Pruszczynski M, de Rooy JW, Veth RP. The cryosurgical treatment of chondroblastoma of bone: long-term oncologic and functional results. J Surg Oncol 2007;96(3):230-4. doi: 10.1002/jso.20804.

[11] Ogura K, Uehara K, Akiyama T, et al. Development of a patient-oriented disease specific outcome measure of health-related quality of life (HRQOL) for musculoskeletal oncology patients. J Orthop Sci 2019;24(3):539-547. doi: 10.1016/j.jos.2018.10.020.

[12] Pritsch T, Bickels J, Wu CC, Squires HM, Malawer MM. The risk for fractures after curettage and cryosurgery around the knee. Clin Orthop Relat Res 2007;458:159-67. doi: 10.1097/BLO.0b013e318038fc3d.

[13] Aversa Z, Costelli P, Muscaritoli M. Cancer-induced muscle wasting: latest findings in prevention and treatment. 
Ther Adv Med Oncol 2017;9(5):369-382. doi: $10.1177 / 1758834017698643$.

[14] Higuchi T, Yamamoto N, Nishida $\mathrm{H}$, et al. Knee joint preservation surgery in osteosarcoma using tumour-bearing bone treated with liquid nitrogen. Int Orthop 2017;41(10):2189-2197. doi: 10.1007/s00264-017-3499-x.

[15] Rowe PJ, Myles CM, Walker C, Nutton R. Knee joint kinematics in gait and other functional activities measured using flexible electrogoniometry: how much knee motion is sufficient for normal daily life? Gait Posture 2000;12(2):143-55. doi: 10.1016/s09666362(00)00060-6.

[16] Schmitt AC, Repka CP, Heise GD, Challis JH, Smith JD. Comparison of posture and balance in cancer survivors and age-matched controls. Clin Biomech (Bristol, Avon) 2017;50:1-6. doi: 10.1016/j.clinbiomech.2017.09.010.

[17] da Silva Alves R, Iunes DH, Pereira IC, Borges JBC, Prado Mariano KO, Carvalho LC. Correlation Between the Trajectory of the Center of Pressure and Thermography of Cancer Patients Undergoing Chemotherapy. J Chiropr Med 2019;18(3):180-187. doi: 10.1016/j.jcm.2019.09.002.

[18] Matsusaka RSHF. New Developments for Limb Salvage in Musculoskeletal Tumors: Springer, 1989.
[19] Calman KC. Quality of life in cancer patients--an hypothesis. J Med Ethics 1984;10(3):124-7.

[20] Huang J, Bi W, Han G, Jia J, Xu M, Wang W. The multidisciplinary treatment of osteosarcoma of the proximal tibia: a retrospective study. BMC Musculoskelet Disord 2018;19(1):315. doi: 10.1186/s12891-0182245-x.

[21] Nayak MG, George A, Vidyasagar MS, et al. Quality of Life among Cancer Patients. Indian J Palliat Care 2017;23(4):445-450.

Conflitos de interesse: Os autores declararam não haver nenhum conflito de interesse.

Agradecimentos: Agradecimento a Fisioterapeuta Andréia Basta pelas orientações e ajuda na realização do trabalho.

Suporte financeiro: Os autores declararam não haver suporte financeiro de terceiros para realização do estudo.

Como citar este artigo: Silva ACAR, Silva FT, Basta A. Análise de força e funcionalidade em paciente oncológico submetido ao criocongelamento: um relato de caso. Brazilian Journal of Case Reports. 2022 Jan-Mar;02(1):47-58. 